\title{
WEAK AND EXACT DOMINATION IN DISTRIBUTED SYSTEMS
}

\author{
LARbi AFIFI* , El Mostafa MAGRI* , AbDelhaq EL JAI** \\ ${ }^{*}$ Faculty of Sciences Ain Chock \\ Hassan II Casablanca University, B.P. 5366-Maârif, Casablanca, Morocco \\ e-mail: larbi_afifi@yahoo.fr,l.afifi@fsac.ac.ma,magrielmostafa@hotmail.com \\ ${ }^{* *}$ IMAGES—Systems Theory \\ University of Perpignan, 52 Avenue Paul Alduy, Perpignan Cedex, France \\ e-mail:aej@univ-perp.fr
}

\begin{abstract}
In this work, we introduce and examine the notion of domination for a class of linear distributed systems. This consists in studying the possibility to make a comparison between input or output operators. We give the main algebraic properties of such relations, as well as characterizations of exact and weak domination. We also study the case of actuators, and various situations are examined. Applications and illustrative examples are also given. By duality, we extend this study to observed systems. We obtain similar results and properties, and the case of sensors is equally examined.
\end{abstract}

Keywords: distributed systems, domination, actuators, sensors.

\section{Introduction}

Controllability and observability are two dual notions which are very important in systems theory and where the choice of input and output operators plays a major role. In this area, the literature is very rich. Characterization results on the notions of weak and exact controllability and observability, as well as strategic actuators and sensors, are established (Afifi and El Jai, 1994; 1995; Afifi et al., 2002a; 2002b; 2008a; Berrahmoune, 1984; Curtain and Pritchard, 1978; Curtain and Zwart, 1995; El Jai and Pritchard, 1987; Jacob and Zwart, 2001; Lions, 1968; 1988; Qarai et al., 2008; Uciński and Korbicz, 1990; Zerrik, 1993; Zerrik et al., 2007). But in almost all these works, the problems considered are focused on the possibility to reach a desired state or reconstruct the state of the examined system, i.e., on studying if a system is (or not) controllable or observable, but without comparison between the input or output parameters themselves.

This work concerns control and observation operator domination for a class of distributed systems. It can be considered as a natural extension of previous works on the notions of controllability and observability.

We consider, without loss of generality, a linear distributed system described by the following state equation:

$$
\text { (S) }\left\{\begin{array}{l}
\dot{z}(t)=A z(t)+B u(t), \\
z(0)=z_{0},
\end{array}\right.
$$

where $A$ generates a strongly continuous semi-group (s.c.s.g.) $(S(t))_{t \geq 0}, B \in \mathcal{L}(U, Z), U$ and $Z$ are respectively the control and state (Hilbert) spaces.

The system (1) is augmented by the following output equation:

$$
y(t)=C z(t),
$$

where $C \in \mathcal{L}(Z, Y), Y$ is the observation space (a Hilbert space). The state $z(t)$ of the system at time $t$ is given by

$$
z(t)=S(t) z_{0}+H_{t}(B) u
$$

where

$$
H_{t}(B) u=\int_{0}^{t} S(t-s) B u(s) \mathrm{d} s
$$

and the observation by

$$
y(t)=C S(t) z_{0}+C H_{t}(B) u .
$$

For the controlled system (1), we study the behavior of the reached space $\operatorname{Im}\left(H_{T}(B)\right)$ with respect to the operator $B$ and the possibility of comparing input operators or actuators.

Concerning observed systems, similar relations are introduced for the output operators or sensors. 
This work is organized as follows: In Section 2, we introduce and characterize the exact and weak dominations. Specific properties of these notions are presented. The case of systems excited by actuators and various situations are also examined. Then, in Section 3, we give an application to a diffusion system and illustrative examples. In the last section, we introduce similar notions for observed systems. By a duality result, we deduce, from the previous sections, analogous results and properties.

Let us note that, with a convenient choice of control and observation spaces, the obtained results can be extended to the case where the operators $B$ and $C$ are unbounded.

\section{Case of controlled systems}

2.1. Problem statement and definitions. We consider the following linear distributed systems:

$$
\begin{aligned}
& \left(S_{1}\right)\left\{\begin{array}{l}
\dot{z}_{1}(t)=A z_{1}(t)+B_{1} u_{1}(t), \quad 0<t<T, \\
z_{1}(0)=z_{1,0} \in Z,
\end{array}\right. \\
& \left(S_{2}\right)\left\{\begin{array}{l}
\dot{z}_{2}(t)=A z_{2}(t)+B_{2} u_{2}(t), \quad 0<t<T, \\
z_{2}(0)=z_{2,0} \in Z,
\end{array}\right.
\end{aligned}
$$

where $A$ is a linear operator generating a strongly continuous semi-group $(S(t))_{t>0}$ on the state space $Z, B_{1} \in$ $\mathcal{L}\left(U_{1}, Z\right), B_{2} \in \mathcal{L}\left(U_{2}, Z\right), u_{1} \in L^{2}\left(0, T ; U_{1}\right)$ and $u_{2} \in$ $L^{2}\left(0, T ; U_{2}\right) ; U_{1}$ and $U_{2}$ are two control spaces (Hilbert spaces).

Without loss of generality, one can assume that $z_{1,0}=z_{2,0}=z_{0}$. In this case, the states of the systems $\left(S_{1}\right)$ and $\left(S_{2}\right)$ at the final time $T$ are respectively given by

$$
\begin{aligned}
& z_{1}(T)=S(T) z_{0}+H_{1} u_{1}, \\
& z_{2}(T)=S(T) z_{0}+H_{2} u_{2},
\end{aligned}
$$

where $H_{1}$ and $H_{2}$ are the operators defined by

$$
H_{1}:\left\{\begin{aligned}
L^{2}\left(0, T ; U_{1}\right) & \longrightarrow Z, \\
u_{1} & \longrightarrow \int_{0}^{T} S(T-s) B_{1} u_{1}(s) \mathrm{d} s,
\end{aligned}\right.
$$

and

$$
H_{2}:\left\{\begin{aligned}
L^{2}\left(0, T ; U_{2}\right) & \longrightarrow Z, \\
u_{2} & \longrightarrow \int_{0}^{T} S(T-s) B_{2} u_{2}(s) \mathrm{d} s .
\end{aligned}\right.
$$

\section{Definition 1.}

1. We say that the system $\left(S_{1}\right)$ dominates $\left(S_{2}\right)$ (or that $B_{1}$ dominates $\left.B_{2}\right)$ exactly on $[0, T]$, if $\operatorname{Im}\left(H_{2}\right) \subset \operatorname{Im}\left(H_{1}\right)$. 2. We say that the system $\left(S_{1}\right)$ dominates $\left(S_{2}\right)$ (or that $B_{1}$ dominates $B_{2}$ ) weakly on $[0, T]$, if $\overline{\operatorname{Im} H_{2}} \subset \overline{\operatorname{Im} H_{1}}$.
In these situations, we write, respectively, $\left(S_{2}\right) \leq$ $\left(S_{1}\right)$ (or $\left.B_{2} \leq B_{1}\right)$ and $\left(S_{2}\right) \preceq\left(S_{1}\right)$ (or $B_{2} \preceq B_{1}$ ).

\section{Remark 1.}

1. Let us note that the relation of exact or weak domination for systems having the same dynamics $A$ is transitive, reflexive but not symmetric, nor antisymmetric. Hence, for example, for any operator $B \neq 0$, we have $\operatorname{Im} B=$ $\operatorname{Im}(2 B)$, even if $B \neq 2 B$.

However, in exact as well as weak cases, one can define equivalence relations (and hence equivalence classes) respectively as follows:

$$
B_{1} \mathcal{R}_{\text {ex }} B_{2} \Longleftrightarrow \operatorname{Im}\left(H_{1}\right)=\operatorname{Im}\left(H_{2}\right)
$$

and

$$
B_{1} \mathcal{R}_{w e} B_{2} \Longleftrightarrow \overline{\operatorname{Im}\left(H_{1}\right)}=\overline{\operatorname{Im}\left(H_{2}\right)} .
$$

2. Exact domination implies weak one. The converse is not true (this will be shown later).

3 . In the case where $\left(S_{1}\right)$ is exactly (respectively, weakly) controllable on $[0, T]$, i.e., $\operatorname{Im} H_{1}=Z$ (resp. $\overline{\operatorname{Im} H_{1}}=Z$ ), then for any operator $B_{2}$, the system $\left(S_{2}\right)$ is dominated exactly (resp. weakly) by $\left(S_{1}\right)$.

4. In fact, one can also consider a single system (S) with two inputs as follows:

$(S)\left\{\begin{array}{l}\dot{z}(t)=A z(t)+B_{1} u_{1}(t)+B_{2} u_{2}(t), \quad 0<t<T \\ z(0)=z_{0} .\end{array}\right.$

In this case, domination concerns only the control operators $B_{1}$ and $B_{2}$. The definitions and all the results remain practically the same.

5. In this work, we consider systems with the same dynamics $A$, but the obtained results can be extended easily to the case where the systems $\left(S_{1}\right)$ and $\left(S_{2}\right)$ have respectively dynamics $A_{1}$ and $A_{2}$, generating, on the state space $Z$, strongly continuous semi-groups $\left(S_{1}(t)\right)_{t \geq 0}$ and $\left(S_{2}(t)\right)_{t \geq 0}$.

2.2. Characterizations. We have the following characterization result concerning the exact domination.

Proposition 1. The following properties are equivalent: (a) The system $\left(S_{1}\right)$ dominates $\left(S_{2}\right)$ exactly on $[0, T]$. (b) For any $u_{2} \in L^{2}\left(0, T ; U_{2}\right)$, there exists a control $u_{1} \in$ $L^{2}\left(0, T ; U_{1}\right)$ such that

$$
H_{1} u_{1}+H_{2} u_{2}=0
$$

(c) There exists $\gamma>0$ such that, for any $\theta \in Z$, we have

$$
\begin{aligned}
\left\|B_{2}^{*} S^{*}(T-\cdot) \theta\right\|_{L^{2}\left(0, T ; U_{2}\right)} & \\
& \leq \gamma\left\|B_{1}^{*} S^{*}(T-\cdot) \theta\right\|_{L^{2}\left(0, T ; U_{1}\right)}
\end{aligned}
$$


Proof. It follows from the definition and from the fact that, if $X, Y$ and $Z$ are reflexive Banach spaces, $P \in \mathcal{L}(X, Z)$ and $Q \in \mathcal{L}(Y, Z)$, then (Curtain and Pritchard, 1978)

$$
\operatorname{Im}(P) \subset \operatorname{Im}(Q)
$$

if and only if there exists $\gamma>0$ such that, for any $z^{*} \in Z^{\prime}$, we have

$$
\left\|P^{*} z^{*}\right\|_{X^{\prime}} \leq \gamma\left\|Q^{*} z^{*}\right\|_{Y^{\prime}}
$$

We give hereafter a characterization of weak (or approximate) domination.

Proposition 2. $\left(S_{1}\right)$ dominates $\left(S_{2}\right)$ weakly, if and only if

$$
\operatorname{ker}\left(B_{1}^{*} S^{*}(\cdot)\right) \subset \operatorname{ker}\left(B_{2}^{*} S^{*}(\cdot)\right)
$$

Proof. It results from the definition and the fact that $\overline{\operatorname{Im} H_{2}} \subset \overline{\operatorname{Im} H_{1}}$ if and only if $\operatorname{ker}\left(H_{1}\right)^{*} \subset \operatorname{ker}\left(H_{2}\right)^{*}$.

2.3. Case of actuators. In what follows, we assume that $Z=L^{2}(\Omega)$ and that $A$ is self-adjoint and has a complete orthonormal system of eigenfunctions $\left\{\varphi_{n j}, j=\right.$ $\left.1, \ldots, r_{n} ; n \in \mathbb{N}^{*}\right\}$ associated with the real eigenvalues $\left(\lambda_{n}\right)_{n \geq 1}$ such that $\lambda_{1}>\lambda_{2}>\lambda_{3}>\ldots$, where $r_{n}$ is the multiplicity of $\lambda_{n}$. In this case,

$$
A z=\sum_{n=1}^{+\infty} \lambda_{n} \sum_{j=1}^{r_{n}}\left\langle z, \varphi_{n j}\right\rangle \varphi_{n j}
$$

and the s.c.s.g. $(S(t))_{t \geq 0}$ generated by $A$ is given by

$$
S(t) z=\sum_{n=1}^{+\infty} e^{\lambda_{n} t} \sum_{j=1}^{r_{n}}\left\langle z, \varphi_{n j}\right\rangle \varphi_{n j}
$$

In the case where $\left(S_{1}\right)$ is excited by $p$ zone actuators $\left(\Omega_{i}, g_{i}\right)_{1 \leq i \leq p}$, we have $U_{1}=\mathbb{R}^{p}$ and (Berrahmoune, 1984; El Jai and Pritchard, 1987)

$$
B_{1}:\left\{\begin{array}{lll}
\mathbb{R}^{p} & \longrightarrow L^{2}(\Omega), \\
\alpha & \longrightarrow & B \alpha=\sum_{i=1}^{p} \alpha_{i} g_{i}
\end{array}\right.
$$

where $\alpha=\left(\alpha_{1}, \ldots, \alpha_{p}\right)^{\operatorname{tr}} \in \mathbb{R}^{p}$ and $g_{i} \in L^{2}(\Omega) ; \Omega_{i}=$ $\operatorname{supp}\left(g_{i}\right) \subset \Omega$. We have

$$
B_{1}^{*} z=\left(\left\langle g_{1}, z\right\rangle, \ldots,\left\langle g_{p}, z\right\rangle\right)^{t r} .
$$

Analogously, if $\left(S_{2}\right)$ is excited by $q$ zone actuators $\left(D_{i}, h_{i}\right)_{1 \leq i \leq q}$, we have $U_{1}=\mathbb{R}^{q}$ and

$$
B_{2}:\left\{\begin{array}{lll}
\mathbb{R}^{q} & \longrightarrow & L^{2}(\Omega) \\
\beta & \longrightarrow & B_{2} \beta=\sum_{i=1}^{q} \beta_{i} g_{i}
\end{array}\right.
$$

with $\beta=\left(\beta_{1}, \ldots, \beta_{q}\right)^{\operatorname{tr}} \in \mathbb{R}^{q}, h_{i} \in L^{2}(\Omega), D_{i}=$ $\operatorname{supp}\left(h_{i}\right) \subset \Omega$ and

$$
B_{2}^{*} z=\left(\left\langle h_{1}, z\right\rangle, \ldots,\left\langle h_{q}, z\right\rangle\right)^{\mathrm{tr}} .
$$

We have the following characterization of the exact domination, resulting from Proposition 1 .

Proposition 3. The system $\left(S_{1}\right)$ dominates $\left(S_{2}\right)$ exactly if and only if there exists $\gamma>0$ such that, for any $z \in Z=$ $L^{2}(\Omega)$, we have

$$
\begin{aligned}
& \left\|\left(\sum_{n=1}^{+\infty} e^{\lambda_{n} t} \sum_{j=1}^{r_{n}}\left\langle z, \varphi_{n j}\right\rangle\left\langle h_{i}, \varphi_{n j}\right\rangle\right)_{1 \leq i \leq q}\right\|_{L^{2}\left(0, T ; \mathbb{R}^{q}\right)} \\
& \leq \gamma\left\|\left(\sum_{n=1}^{+\infty} e^{\lambda_{n} t} \sum_{j=1}^{r_{n}}\left\langle z, \varphi_{n j}\right\rangle\left\langle g_{i}, \varphi_{n j}\right\rangle\right)_{1 \leq i \leq p}\right\|_{L^{2}\left(0, T ; \mathbb{R}^{p}\right)} .
\end{aligned}
$$

We give hereafter a necessary condition concerning exact domination.

Proposition 4. If the system $\left(S_{1}\right)$ dominates $\left(S_{2}\right)$ exactly, then there exists a real $\gamma>0$ such that, for any $n \in \mathbb{N}^{*}$ and $j \in\left\{1, \ldots, r_{n}\right\}$, we have

$$
\left\|\left(\left\langle h_{i}, \varphi_{n j}\right\rangle\right)_{1 \leq i \leq q}\right\|_{\mathbb{R}^{q}} \leq \gamma\left\|\left(\left\langle g_{i}, \varphi_{n j}\right\rangle\right)_{1 \leq i \leq p}\right\|_{\mathbb{R}^{p}} .
$$

Proof. Using the previous proposition, if $\left(S_{1}\right)$ dominates $\left(S_{2}\right)$ exactly, then there exists $\gamma>0$ satisfying the inequality (17) for any $z \in Z$. For $z=\varphi_{n j}$ in this inequality, we obtain

$$
\begin{aligned}
& \left\|e^{\lambda_{n} t}\left(\left\langle h_{i}, \varphi_{n j}\right\rangle\right)_{1 \leq i \leq q}\right\|_{L^{2}(0, T ; \mathbb{R} q)} \\
& \quad \leq \gamma\left\|\left(e^{\lambda_{n} t}\left\langle g_{i}, \varphi_{n j}\right\rangle\right)_{1 \leq i \leq p}\right\|_{L^{2}\left(0, T ; \mathbb{R}^{p}\right)}
\end{aligned}
$$

or, equivalently,

$$
\begin{aligned}
& \left\|e^{\lambda_{n} t}\right\|_{L^{2}(0, T ; \mathbb{R})}\left\|\left(\left\langle h_{i}, \varphi_{n j}\right\rangle\right)_{1 \leq i \leq q}\right\|_{\mathbb{R}^{q}} \\
& \quad \leq \gamma\left\|e^{\lambda_{n} t}\right\|_{L^{2}(0, T ; \mathbb{R})}\left\|\left(\left\langle g_{i}, \varphi_{n j}\right\rangle\right)_{1 \leq i \leq p}\right\|_{\mathbb{R}^{p}} .
\end{aligned}
$$

Since $\left\|e^{\lambda_{n} t}\right\|_{L^{2}(0, T ; \mathbb{R})}>0$, we have

$$
\left\|\left(\left\langle h_{i}, \varphi_{n j}\right\rangle\right)_{1 \leq i \leq q}\right\|_{\mathbb{R}^{q}} \leq \gamma\left\|\left(\left\langle g_{i}, \varphi_{n j}\right\rangle\right)_{1 \leq i \leq p}\right\|_{\mathbb{R}^{p}} .
$$

An immediate consequence of Proposition 4 is that if

$$
\left\langle g_{i}, \varphi_{n_{0} j}\right\rangle=0, \quad 1 \leq i \leq p
$$

for some $n_{0} \in \mathbb{N}^{*}$ and $j \in\left\{1, \ldots, r_{n_{0}}\right\}$, then

$$
\left\langle h_{i}, \varphi_{n_{0} j}\right\rangle=0, \quad 1 \leq i \leq q
$$

In some situations, this can be used to show that (or to reconstruct) a system (which) is not dominated exactly by another one. Concerning weak domination, we give the following definition. 
Definition 2. If $B_{2} \preceq B_{1}$, we say that $\left(D_{i}, g_{i}\right)_{1<i<p}$ are more strategic than $\left(\bar{\Omega}_{j}, h_{j}\right)_{1 \leq j \leq q}$.

Now, let us show the following result, which leads to matrix characterization of weak domination.

Lemma 1. We have

$$
\begin{aligned}
& \operatorname{ker} B_{1}^{*} S^{*}(\cdot) \\
& \quad=\left\{z \in Z \mid\left(\left\langle z, \varphi_{n j}\right\rangle\right)_{j=1, r_{n}} \in \operatorname{ker} M_{n}, \forall n \geq 1\right\}
\end{aligned}
$$

where $M_{n}$ is the controllability matrix defined by

$$
M_{n}=\left(\left\langle g_{i}, \varphi_{n j}\right\rangle\right)_{1 \leq i \leq p ; 1 \leq j \leq r_{n}} .
$$

Proof. We have

$$
B_{1}^{*} S^{*}(t) z=\left(\sum_{n=1}^{+\infty} e^{\lambda_{n} t} \sum_{j=1}^{r_{n}}\left\langle z, \varphi_{n j}\right\rangle\left\langle g_{i}, \varphi_{n j}\right\rangle\right)_{1 \leq i \leq p}
$$

Then $z \in \operatorname{ker} B_{1}^{*} S^{*}(\cdot)$ if and only if

$$
\sum_{n=1}^{+\infty} e^{\lambda_{n} t} \sum_{j=1}^{r_{n}}\left\langle z, \varphi_{n j}\right\rangle\left\langle g_{i}, \varphi_{n j}\right\rangle=0, \forall i \in\{1, \ldots, n\} .
$$

Using the analyticity property, this is equivalent to

$$
\sum_{j=1}^{r_{n}}\left\langle z, \varphi_{n j}\right\rangle\left\langle g_{i}, \varphi_{n j}\right\rangle=0
$$

$$
\forall n \geq 1, \forall i \in\{1, \ldots, p\}
$$

i.e.,

$$
\forall n \in \mathbb{N}^{*},\left(\left\langle z, \varphi_{n j}\right\rangle\right)_{1 \leq j \leq r_{n}} \in \operatorname{ker} M_{n}
$$

It is well known that the actuators $\left(D_{i}, g_{i}\right)_{1 \leq i \leq p}$ are strategic (i.e., the corresponding system $\left(S_{1}\right)$ is weakly controllable) if and only if

$$
\operatorname{rank}\left(M_{n}\right)=r_{n}, \quad \forall n \geq 1 .
$$

We have the same results concerning $\left(S_{2}\right)$ by replacing $M_{n}$ with the matrix $G_{n}$ defined by

$$
G_{n}=\left(\left\langle h_{i}, \varphi_{n j}\right\rangle\right)_{1 \leq i \leq q ; 1 \leq j \leq r_{n}} .
$$

The following proposition gives a necessary and sufficient condition for weak domination.

Proposition 5. The system $\left(S_{1}\right)$ dominates $\left(S_{2}\right)$ weakly if and only if

$$
\operatorname{ker} M_{n} \subset \operatorname{ker} G_{n}, \quad \forall n \in \mathbb{N}^{*} \text {. }
$$

Proof. Assume that $\operatorname{ker} M_{n} \subset \operatorname{ker} G_{n}$, for any $n \in \mathbb{N}^{*}$. Using Lemma1, we have $z \in \operatorname{ker} B_{1}^{*} S^{*}(\cdot)$

$$
\begin{aligned}
& \Leftrightarrow \forall n \in \mathbb{N}^{*},\left(\left\langle z, \varphi_{n j}\right\rangle\right)_{j=1, r_{n}} \in \operatorname{ker} M_{n} \\
& \Rightarrow \forall n \in \mathbb{N}^{*},\left(\left\langle z, \varphi_{n j}\right\rangle\right)_{j=1, r_{n}} \in \operatorname{ker} G_{n} \\
& \Rightarrow z \in \operatorname{ker}\left(B_{2}^{*} S^{*}(\cdot)\right) .
\end{aligned}
$$

Conversely, if

$\exists m \in \mathbb{N}^{*}$ such that $\operatorname{ker} M_{m} \not \subset \operatorname{ker} G_{m}$,

then there exist $\left(\alpha_{m j}\right)_{1 \leq j \leq r_{m}} \in \mathbb{R}^{r_{m}}$ which satisfy $\left(\alpha_{m j}\right)_{1 \leq j \leq r_{m}} \in \operatorname{ker} M_{m} \backslash \operatorname{ker} G_{m}$. For

$$
z=\sum_{j=1}^{r_{n}} \alpha_{m j} \varphi_{m j}
$$

we have

$$
B_{1}^{*} S^{*}(t) z=\left(e^{\lambda_{m} t} \sum_{j=1}^{r_{m}} \alpha_{n j}\left\langle g_{i}, \varphi_{m j}\right\rangle\right)_{1 \leq i \leq p}=0_{\mathbb{R}^{p}}
$$

and

$$
B_{2}^{*} S^{*}(t) z=\left(e^{\lambda_{m} t} \sum_{j=1}^{r_{m}} \alpha_{n j}\left\langle h_{i}, \varphi_{m j}\right\rangle\right)_{1 \leq i \leq q} \neq 0_{\mathbb{R}^{q}} .
$$

Consequently,

$$
\operatorname{ker}\left(B_{1}^{*} S^{*}(\cdot)\right) \not \subset \operatorname{ker}\left(B_{2}^{*} S^{*}(\cdot)\right) .
$$

The above result is a practical tool to study weak domination and then to make a comparison between actuators using Definition 2. Now, we give hereafter other specific properties and characteristics concerning the notions of weak and exact domination.

\section{Proposition 6.}

(P1) There exist operators $B_{1}$ and $B_{2}$ such that any one of them do not dominate the other.

(P2) An actuator $(\bar{D}, h)$ may be dominated weakly by a non strategic actuator $(D, g)$.

(P3) An actuator $(D, g)$ may dominate another actuator $(\bar{D}, h)$ weakly, but not exactly.

(P4) A system excited by $p$ actuators (with $p>1$ ) may be dominated exactly or weakly by another system excited by one actuator (and hence by $q$ actuators with $q<p$ ).

These properties and situations are illustrated in the following section 


\section{Application to a diffusion system and illustrative examples}

We consider, without loss of generality, the onedimensional case where $\Omega=] 0, a[$ and where the systems $\left(S_{1}\right)$ and $\left(S_{2}\right)$ are respectively described by the following equations:

$$
\left(S_{1}\right)\left\{\begin{array}{rlr}
\frac{\partial z_{1}}{\partial t}(x, t) & =\frac{\partial^{2} z_{1}}{\partial x^{2}}(x, t) & \\
& +g(x) u(t), \quad \Omega \times] 0, T[, \\
z_{1}(0, t) & \left.=z_{1}(a, t)=0,\right] 0, T[, \\
z_{1}(x, 0) & =0, & ] 0, a[,
\end{array}\right.
$$

and

$$
\left(S_{2}\right)\left\{\begin{array}{rlr}
\frac{\partial z_{2}}{\partial t}(x, t)= & \frac{\partial^{2} z_{2}}{\partial x^{2}}(x, t) \\
& +h(x) v(t), \quad \Omega \times] 0, T[, \\
z_{2}(0, t)= & \left.z_{2}(a, t)=0,\right] 0, T[ \\
z_{2}(x, 0) & =0, & ] 0, a[.
\end{array}\right.
$$

In this case, we have $Z=L^{2}(\Omega)$ and $A z=\Delta z$ for $z \in D(A)=H^{2}(\Omega) \cap H_{0}^{1}(\Omega)$. $A$ yields a complete system of eigenfunctions $\left(\varphi_{n}\right)_{n \in \mathbb{N}^{*}}$ associated with the eigenvalues

$$
\lambda_{n}=-\frac{n^{2} \pi^{2}}{a^{2}}
$$

with

$$
\varphi_{n}(x)=\sqrt{\frac{2}{a}} \sin \frac{n \pi x}{a} .
$$

First, let us recall that an actuator $(\Omega, g)$ is strategic if and only if, for any $n \geq 1$, we have

$$
\left\langle g, \varphi_{n}\right\rangle \neq 0 .
$$

Obviously, a strategic actuator dominates weakly any finite number of other actuators. We also note that $(\Omega, g)$ dominate $(\Omega, h)$ weakly if, and only if, for any $n \in \mathbb{N}^{*}$, we have

$$
\left\langle g, \varphi_{n}\right\rangle=0 \Longrightarrow\left\langle h, \varphi_{n}\right\rangle=0 .
$$
ples.

Property (P1) is illustrated by the following exam-

Example 1. Let $m, n \in \mathbb{N}^{*}$ such that $m \neq n$. We suppose that $\left(S_{1}\right)$ is excited by an actuator $(\Omega, g)$ with a spatial distribution $g=\varphi_{n}$ and that $\left(S_{2}\right)$ is excited by an actuator $(\Omega, h)$ with a spatial distribution $h=\varphi_{m}$. For $p=m+n$, we have

$$
\left\langle\varphi_{m}, \varphi_{p}\right\rangle=\left\langle\varphi_{n}, \varphi_{p}\right\rangle=0 .
$$

Then these actuators are not strategic. Moreover,

$$
\left\langle g, \varphi_{n}\right\rangle=1, \quad\left\langle g, \varphi_{m}\right\rangle=0
$$

and

$$
\left\langle h, \varphi_{n}\right\rangle=0, \quad\left\langle h, \varphi_{m}\right\rangle=1 .
$$

Then any one of these actuators does not dominate the other weakly.

Example 2. For $g(x)=x(a-x)$, we have

$$
\left\langle g, \varphi_{n}\right\rangle=\int_{0}^{a} \sqrt{\frac{2}{a}}(a-x) x \sin \left(\frac{\pi x n}{a}\right) \mathrm{d} x
$$

With $t=\pi x / a$, we have

$$
\left\langle g, \varphi_{n}\right\rangle=\sqrt{\frac{2}{a}}\left(\frac{a}{\pi}\right)^{3} \int_{0}^{\pi}(\pi-t) t \sin (n t) \mathrm{d} t .
$$

Using integration by parts, we obtain

$$
\begin{aligned}
\int_{0}^{\pi} & P(t) \sin (n t) \mathrm{d} t \\
= & {\left[-P(t) \frac{\cos n t}{n}+P^{\prime}(t) \frac{\sin n t}{n^{2}} P^{\prime \prime}(t) \frac{\cos n t}{n^{3}}\right]_{0}^{\pi} } \\
& -\int_{0}^{\pi} P^{(3)}(t) \frac{\cos n t}{n^{3}} \mathrm{~d} t
\end{aligned}
$$

Then

$$
\left\langle g, \varphi_{n}\right\rangle=\sqrt{\frac{2}{a}}\left(\frac{a}{\pi n}\right)^{3} 2\left(1-(-1)^{n}\right) .
$$

For $h=2 x-a$, we have

$$
\left\langle h, \varphi_{n}\right\rangle=\int_{0}^{a} \sqrt{\frac{2}{a}}(2 x-a) \sin \left(\frac{\pi x n}{a}\right) \mathrm{d} x .
$$

We obtain, by the same token,

$$
\left\langle h, \varphi_{n}\right\rangle=-\sqrt{\frac{2}{a}} \frac{a^{2}\left(1+(-1)^{n}\right)}{\pi n} .
$$

Then

$$
\forall n \in \mathbb{N}^{*},\left\{\begin{array}{l}
\left\langle g, \varphi_{n}\right\rangle=0 \Longleftrightarrow n=2 p(p \geq 1), \\
\left\langle g, \varphi_{n}\right\rangle=0 \Longleftrightarrow n=2 p+1(p \geq 0) .
\end{array}\right.
$$

Consequently, $(\Omega, g)$ and $(\Omega, h)$ are not strategic and any one of them does not dominate the other weakly.

Now, in order to illustrate Property (P2), we consider the following situation.

Example 3. For $\bar{D}=D=\Omega ; g=\varphi_{1}+\varphi_{2}$ and $h=\varphi_{1}$, we have

$$
\forall n \in \mathbb{N}^{*},\left\langle g, \varphi_{n}\right\rangle=0 \Longrightarrow n \geq 3 \Longrightarrow\left\langle h, \varphi_{n}\right\rangle=0 .
$$

Then $(\Omega, g)$ is not strategic and $(\Omega, g)$ dominate $(\Omega, h)$ weakly. Moreover, one can also verify that $(\Omega, g)$ dominate $(\Omega, h)$ exactly. 
Concerning (P3), we consider the following example.

Example 4. For $\bar{D}=D=\Omega ; g=x\left(x^{2}-a^{2}\right)$ and $h=2 x-a$, we have

$$
\left\langle g, \varphi_{n}\right\rangle=\int_{0}^{a} \sqrt{\frac{2}{a}} x\left(x^{2}-a^{2}\right) \sin \left(\frac{n \pi x}{a}\right) \mathrm{d} x .
$$

For $t=\pi x / a$, we have

$$
\left\langle g, \varphi_{n}\right\rangle=\sqrt{\frac{2}{a}}\left(\frac{a}{\pi}\right)^{4} \int_{0}^{\pi} t\left(t^{2}-\pi^{2}\right) \sin (n t) \mathrm{d} t
$$

Using integration by parts, we obtain

$$
\left\langle g, \varphi_{n}\right\rangle=6 \sqrt{2 a}(-1)^{n}\left(\frac{a}{n \pi}\right)^{3} .
$$

Then $(\Omega, g)$ is strategic. To avoid such a case, we consider

$$
g(x)=x\left(x^{2}-a^{2}\right)+6 \sqrt{2 a}\left(\frac{a}{\pi}\right)^{3} \varphi_{1}(x) .
$$

We have

$$
\left\langle g, \varphi_{1}\right\rangle=0
$$

and

$$
\left\langle g, \varphi_{n}\right\rangle=6 \sqrt{2 a}(-1)^{n}\left(\frac{a}{\pi n}\right)^{3}, \quad \forall n \geq 2 .
$$

On the other hand,

$$
\left\langle h, \varphi_{n}\right\rangle=-\sqrt{\frac{2}{a}} \frac{a^{2}\left(1+(-1)^{n}\right)}{\pi n} .
$$

Since, for every $n \in \mathbb{N}^{*}$, we have that

$$
\left\langle g, \varphi_{n}\right\rangle=0 \Longrightarrow\left\langle h, \varphi_{n}\right\rangle=0,
$$

$(\Omega, g)$ dominate weakly $(\Omega, h)$. Let us show that $(\Omega, g)$ do not dominate $(\Omega, h)$ exactly. sition 4

If $(\Omega, g)$ dominate $(\Omega, h)$ exactly, then, using Propo-

$$
\exists \gamma>0 \text { such that }\left|\left\langle h, \varphi_{n}\right\rangle\right| \leq \gamma\left|\left\langle g, \varphi_{n}\right\rangle\right|, \forall n \in \mathbb{N}^{*} .
$$

For $n=2 p$, we obtain

$$
\sqrt{\frac{2}{a}} \frac{a^{2}}{\pi p} \leq \gamma 6 \sqrt{2 a}\left(\frac{a}{2 \pi p}\right)^{3}, \quad \forall p \in \mathbb{N}^{*} .
$$

which is not possible. Consequently, $(\Omega, g)$ do not dominate $(\Omega, h)$ exactly.

The last property is illustrated hereafter.

Example 5. It is sufficient to consider the case where $B_{1}$ is associated with the actuator $\left([0, a], x\left(x^{2}-a^{2}\right)\right)$ and $B_{2}$ is associated with the two actuators $\left(\left([0, a / 2], \varphi_{2}\right)\right.$ and $\left.\left([a / 2, a], \varphi_{4}\right)\right)$.

\section{Case of observed systems}

In this part, we extend the notion of domination established for input operators and controlled systems, to the case of output operators and observed systems. We introduce the same notion of domination for observed systems and sensors. The characterizations and results are similar to those obtained in the previous section and can be deduced by duality.

Let $Z$ be a separable Hilbert space. We consider the system

$$
\left(S^{\prime}\right)\left\{\begin{array}{l}
\dot{z}(t)=A z(t), \quad 0<t<T \\
z(0)=z_{0}
\end{array}\right.
$$

where $A$ is a linear operator generating a strongly continuous semi-group $(S(t))_{t \geq 0}$.

The system (26) is augmented with the output equations

$$
\begin{array}{ll}
y_{1}(t)=C_{1} z(t), & 0<t<T, \\
y_{2}(t)=C_{2} z(t), & 0<t<T,
\end{array}
$$

where $C_{i} \in \mathcal{L}\left(Z, Y_{i}\right), i=1,2$. Here $Y_{1}$ and $Y_{2}$ are Hilbert spaces (observation spaces).

The first observation is given by

$$
y_{1}(t)=C_{1} S(t) z_{0}, \quad 0<t<T,
$$

and the second one by

$$
y_{2}(t)=C_{2} S(t) z_{0}, \quad 0<t<T .
$$

For $i=1,2$, we have

$$
y_{i}(\cdot)=K_{i}(\cdot) z_{0}
$$

with

$$
K_{i}=C_{i} S(\cdot)
$$

and

$$
K_{i}^{*} y=\int_{0}^{T} S^{*}(t) C_{i}^{*} y(t) \mathrm{d} t .
$$

We introduce the following similar definitions.

\section{Definition 3.}

(i) We say that $C_{1}$ dominates exactly $C_{2}$ on $[0, T]$, with respect to the system $(S)$, if

$$
\operatorname{Im} K_{2} \subset \operatorname{Im} K_{1}
$$

(ii) We say that $C_{1}$ dominates weakly $C_{2}$ on $[0, T]$, with respect to the system $(S)$, if

$$
\overline{\operatorname{Im} K_{2}} \subset \overline{\operatorname{Im} K_{1}}
$$

In these cases, we note respectively $C_{2} \leq C_{1}$ and $C_{2} \preceq C_{1}$. 
Let $C_{i}=B_{i}^{*}$; for $i=1,2$. We consider the dual systems

$$
\left(S_{i}^{*}\right)\left\{\begin{array}{l}
\dot{z}(t)=A^{*} z(t)+B_{i} u_{i}(t), \quad 0<t<T, \\
z(0)=z_{0},
\end{array}\right.
$$

and

$$
\left(\widetilde{S}_{i}\right)\left\{\begin{array}{l}
\dot{z}(t)=A^{*} z(t), \quad 0<t<T, \\
y_{i}(t)=C_{i} z(t) .
\end{array}\right.
$$

For $i=1,2$, it is well known that the system 29] is controllable if and only if (30) is observable. From this duality between the notions of controllability and observability, one can deduce the following characterization result.

Proposition 7. The observed system $\left(\widetilde{S}_{1}\right)$ dominates exactly (respectively weakly) $\left(\widetilde{S}_{2}\right)$, if and only if, the controlled system $\left(S_{1}^{*}\right)$ dominates exactly (respectively weakly) $\left(S_{2}^{*}\right)$.

Using this duality, a general result concerning exact and weak domination for controlled and observed systems, we obtain similar results and properties by replacing $B_{i}$ with $C_{i}^{*}$ and $H_{i}$ with $K_{i}^{*}$, and hence by replacing actuator(s) with sensor(s).

Finally, let us note that in this work we consider a class of linear systems, but the results and the approach considered can be extended to other systems and situations (non-linear systems, delayed systems, unbounded operators, regional case, etc.) .

\section{Acknowledgment}

The authors wish to thank the Academy Hassan II of Sciences and Technics for its support to the Systems Theory Network.

\section{References}

Afifi, L. and El Jai, A. (1994). Strategic sensors and spy sensors, International Journal of Applied Mathematics and Computer Science 4(4): 553-573.

Afifi, L. and El Jai, A. (1995). Spy sensors and detection, International Journal of Systems Science 26(3): 1447-1463.

Afifi, L., Chafiai, A. and Bel Fekih, A. (2002a) Enlarged exact compensation in distributed systems, International Journal of Applied Mathematics and Computer Science 12(4): 467477

Afifi, L., Chafiai, A. and El Jai, A. (2002b). Regionally efficient and strategic actuators, International Journal of Systems Science 33(1): 1-12.

Afifi, L., El Jai, A. and Zerrik, E.H. (2008a). Systèmes Dynamiques II: Analyse régionale des systèmes linéaires distribués, University of Perpignan Press, Perpignan.
Afifi, L., El Jai A. and Magri E. M. (2008b). Compensation problem in finite dimension linear dynamical systems, International Journal of Applied Mathematical Sciences 2(45): 2219-2228.

Balakrishnan, A.V. (1981). Applied Functional Analysis, Springer Verlag, New York, NY.

Berrahmoune, L. (1984). Localisation d'actionneurs pour la contrôlabilité de systèmes paraboliques et hyperboliques. Application par dualité à la localisation de capteurs, Ph.D. thesis, University of Mohammed V, Rabat, Morocco.

Curtain, R.F. and Pritchard, A.J. (1978). Infinite Dimensional Linear Systems Theory, Springer, Berlin.

Curtain, R.F. and Zwart, H.J. (1995). An Introduction to InfiniteDimensional Linear Systems Theory, Springer Verlag, New York, NY.

El Jai, A. and Pritchard, A.J. (1987). Sensors and actuators in distributed systems, International Journal of Control 46(4): 1139-1153.

Jacob, B. and Zwart, H.J. (2001). Exact observability of diagonal systems with a one dimension output operator, International Journal of Applied Mathematics and Computer Science 11(6): 1277-1283.

Klamka, J. (2002). Controllability of linear discrete systems, International Journal of Applied Mathematics and Computer Science 12(2): 173-180.

Lions, J.L. (1968). Contrôle optimal de systèmes gouvernés par des équations aux dérivées partielles, Dunod, Paris.

Lions, J.L. (1988). Contrôlabilité exacte, perturbations et stabilisation des systèmes distribués, Masson, Paris.

Qarai, Y., Bernoussi, A. and El Jai A. (2008). How to compensate a spreading disturbance for a class of nonlinear systems, International Journal of Applied Mathematics and Computer Science 18(2): 171-187, DOI: 10.2478/v10006-0080016-9.

Uciński, D. and Korbicz, J. (1990). Parameter identification of two dimensional distributed systems, International Journal of Systems Science 21(12): 2441-2456.

Zerrik, E. (1993). Regional analysis of distributed parameter systems, Ph.D. thesis, University of Rabat, Morocco.

Zerrik, E., Larhrissi, R. and Bourray, H. (2007). An output controllability problem for semi-linear distributed hyperbolic systems, International Journal of Applied Mathematics and Computer Science 17(4): 437-448, DOI: 10.2478/v10006-007-0035-y.

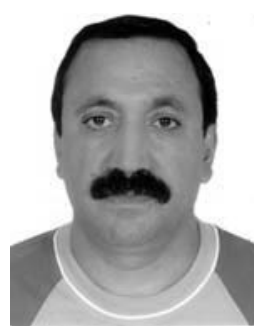

Larbi Afifi is a professor at Hassan II Casablanca University, Faculty of Sciences Ain Chock. His research activities concern essentially the aspects of modeling, analysis and control of distributed parameter systems. He is the head of the Modeling, Analysis and Control of Systems (MACS) research laboratory at Hassan II Casablanca University, Faculty of Sciences Ain Chock, Morocco. 


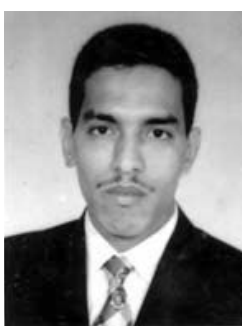

El Mostafa Magri is an associate professor and a member of the MACS laboratory, Hassan II Casablanca University, Faculty of Sciences Ain Chock, Morocco. His research activities concern the analysis and control of dynamical systems, as well as the notions of actuators and sensors in distributed parameter systems.

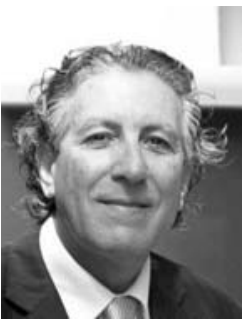

Abdelhaq El Jai is a professor at the University of Perpignan. His activities for more than 30 years in the research area have been concerned with various aspects of modeling, analysis and control in distributed parameter systems. He has been, since 2006, a member of the Academy Hassan II of Sciences and Technics in Morocco. He is also the coordinator of the Systems Theory Network.

Received: 22 July 2009

Revised: 25 January 2010

Re-revised: 4 May 2010 